\title{
CANADIAN ANAESTHETISTS' MUTUAL ACCUMULATING FUND LIMITED
}

The second fiscal year of the Fund ended September 30, 1959, and analysis has shown that performance during that year was at least equal to other Canadian mutual funds, and in most instances much better, despite the market decline of the late summer and autumn. The Board of Directors has continued the policy of no "front-end loading," and no penalty on redemption of shares.

The shares of the Fund are registered for sale to the public in all the provinces of Canada except Prince Edward Island and Newfoundland. Enquiries may be addressed to the Head Office of the Fund at the following address:

THE CANADIAN ANAESTHETISTS' MIUTUAL ACCUMIULATING FUND LTD.

178 St. George St., Toronto 5, Ontario

INTERiR STATEMENT, JANUARY 1, 1960

\footnotetext{
Current

Cash

Accrued interest receivable

Dividends receivable

Investments in marketable securities (at market valuis) (Cost $\$ 355,861.94$ )

Organization expenses
}

Current

Accrued liabilities

Shareholders' deposits for investment

Provision for income tax

Liabilitie's

Assets

Capital stock

Issued fully paid

68,462 Class " $A$ " shares of $\$ 1.00$ each 8 Class " $B$ " shares of $\$ 1.00$ each $\overline{68,470}$

Shareholders' Equity

Reserve for organization expense

Reserve for redemptions

Unrealized appreciation on investments

Realized gain on investments

Surplus

Balance September 30, 1959

Interim net gain October 1, 1959, to January 4, 1960

Value of shares January 4, 1960 :

$\$ 388,752.79+68,470=\$ 5.68$
\$ $\quad 15,755.95$

460.00

$575.00 \quad \& \quad 16,790.95$

$365,128.00$

$s \frac{9,209.75}{391,128.70}$

$5 \quad 1,682.11$

565.33

128.47
$\$ 68,462.00$
${ }^{8.00} 0^{\circ} \$ 68,470.00$
$9,209.75$
$293,858.78$
9,266.06
$5,250.13 \quad 14,516.19$
$1,188.40$
1,509.67
2,698.07
388.752 .79
$\$ 391,128.70$


Securities Heid by the Fund

Bonds

Bicroft Uranium

Cdn. Delhi (conv.)

Faraday Uranium Mines
Greyhound: (conv.)

Milliken Lake

Westcoast (conv.)

\section{SHares}

Preference

B.C. Electric

I.A.C.

Massey-Ferguson

Common

Abitibi Power \& Paper

Algoma Steel Corporation

Imperial cil

Aluminium Limited

Argus Corp.

Bank of Montreal

B.C. Power

Canadian Oil

Consolidated Paper

Consumers' Gas Co.

Dominion Foundries

Dominion Stores

Great Lakes Paper

Greyhound Lines of Canada

Howard Smith Paper Mills

I.A.C.

International Nickel Company

Interprovincial Pipe Line Co.

Mackillan \& Bloedel "B"

Moore Coip.

Noranda Mines Limited

Page-Hersey Tubes

Simpson's Limited

Stedman Bros.

Steel Company of Canada

Superior Propane

Toronto-Dominion Bank

George W'eston Ltd. B 